\title{
Управление состояниями промышленных технологий на основе критерия риска
}

C.P. Бакасов 1, аспиранm, sabir17204@gmail.com

Г.Н. Санаева 2, старший преподаватель, gsanaeva@nirhtu.ru

B.H. Богатиков ${ }^{1}$, д.m.н., npoфpeccop, vnbgtk@mail.ru

${ }_{1}$ Тверской государственный технический университет, г.Тверь, 170026, Россия

${ }^{2}$ Новомосковский институт РХТУ им. Д.И. Менделеева,

2. Новомосковск, 301665, Россия

Работа посвящена исследованию концептуальной постановки задачи управления состояниями промышленных технологий. Рассмотрено управление потенциально опасной технологией селективной очистки хвостовых газов производства неконцентрированной азотной кислоты. Это является приложением идеи управления состояниями. В данном приложении управление состояниями тесно связано с управлением безопасностью промышленных систем.

Одной из проблем синтеза систем управления безопасностью промышленных технологий является наличие неопределенности в знаниях о физико-химических процессах, а также неопределенности, связанной с влиянием случайных возмущений. Это порождает необходимость разработки новых методов синтеза систем управления технологической безопасностью, а также совершенствования существующих систем. Перспективным подходом для такого рода динамических процессов, протекающих в слабоструктурированных и плохо формализуемых средах, являются методы реализации механизмов целеполагания и пересмотра критериев качества управления. Эти методы базируются на фундаментальных знаниях. Различного типа дефекты находят отражение в переменных состояния технологических процессов. Нарушения могут порождаться дефектами систем управления, технологического оборудования, в самом технологическом процессе. Всевозможные повреждения в технологической системе (несоответствие требованиям исходных материалов, несоблюдение требований нормативно-технических документов, человеческий фактор) приводят к сходным результатам. Это говорит о сложности как проведения процедуры диагноза, так и формирования критериев оценки состояний.

С точки зрения управления система обеспечения технологической безопасности является в настоящее время многоуровневой иерархически организованной технологической системой. Основная цель таких систем - своевременное обнаружение неисправностей и принятие мер по устранению их первопричин. В работе рассматривается многоуровневая организация системы обеспечения технологической безопасности.

В качестве приложения к предложенному подходу рассматривается многоуровневая организация системы обеспечения технологической безопасности процесса селективной очистки газов производства слабой азотной кислоты. Предложены основной критерий управления технологий - критерий риска ведения технологического процесса и импульсная модель критерия. Управление строится на основе управления с предсказанием.

Применение разработанной системы позволило не только повысить экономические показатели, но и уменьшить загрязнение воздушного бассейна.

Ключевые слова: диагностика состояний, обеспечение технологической безопасности, риск работы промышленной технологии.

Организация управления системами обеспечения безопасности в промышленных технологиях, которые относятся к классу сложных современных систем, обретает все большую значимость. Принятие решений в данном случае осуществляется в слабоструктурированной и плохо формализуемой среде. Наиболее успешно можно решать такие задачи с помощью интегрального подхода, сочетающего в себе как классическую математику, так и со- временные достижения из области моделей методов искусственного интеллекта. В последнее десятилетие использование этих методов становится все более актуальным, особенно в управлении промышленными системами с целью повышения качества принимаемых решений [1].

Формализованное концептуальное представление оценки технологической безопасности $\boldsymbol{T}_{s}{ }^{k}$ может быть следующим: $\boldsymbol{T}_{s}{ }^{k}=\left\langle\boldsymbol{D}^{k-1}\right.$ 
$\left(\boldsymbol{D}^{k-2}, \ldots\right), \boldsymbol{U}^{k}\left(\boldsymbol{U}^{k-1}, \ldots\right), \boldsymbol{R}, \boldsymbol{B}^{k}\left(\boldsymbol{B}^{k-1}, \ldots\right), \boldsymbol{A}>$, где $k$ - моменты времени оценки состояний; $\boldsymbol{D}^{k}-$ вектор опасностей; $\boldsymbol{U}^{k}-$ вектор управлений, компенсирующих опасности; $\boldsymbol{D} \times \boldsymbol{U}-$ композиции опасностей и управлений; $\boldsymbol{D} \times \boldsymbol{U} \rightarrow \boldsymbol{R}-$ соответствие принимаемых решений. Величина безопасности $\boldsymbol{B}^{k}-$ вектор оценок безопасности, нормированный по некоторому правилу (например, это могут быть точки интервала
$[0,1])$ либо на булевское множество <false, true $>$ (например, $\left.\boldsymbol{B}^{k} \subseteq[0,1]\right) ; \boldsymbol{A}$ - некоторые операторы преобразования, участвующие в оценке уровня безопасности (например, вероятностные, нечеткие). Для реальных процессов промышленной технологии $\boldsymbol{D}$ и $\boldsymbol{U}$ конечны и счетны. Концептуальная модель управления технологической безопасностью приведена на рисунке 1 , а структура системы - на рисунке 2.

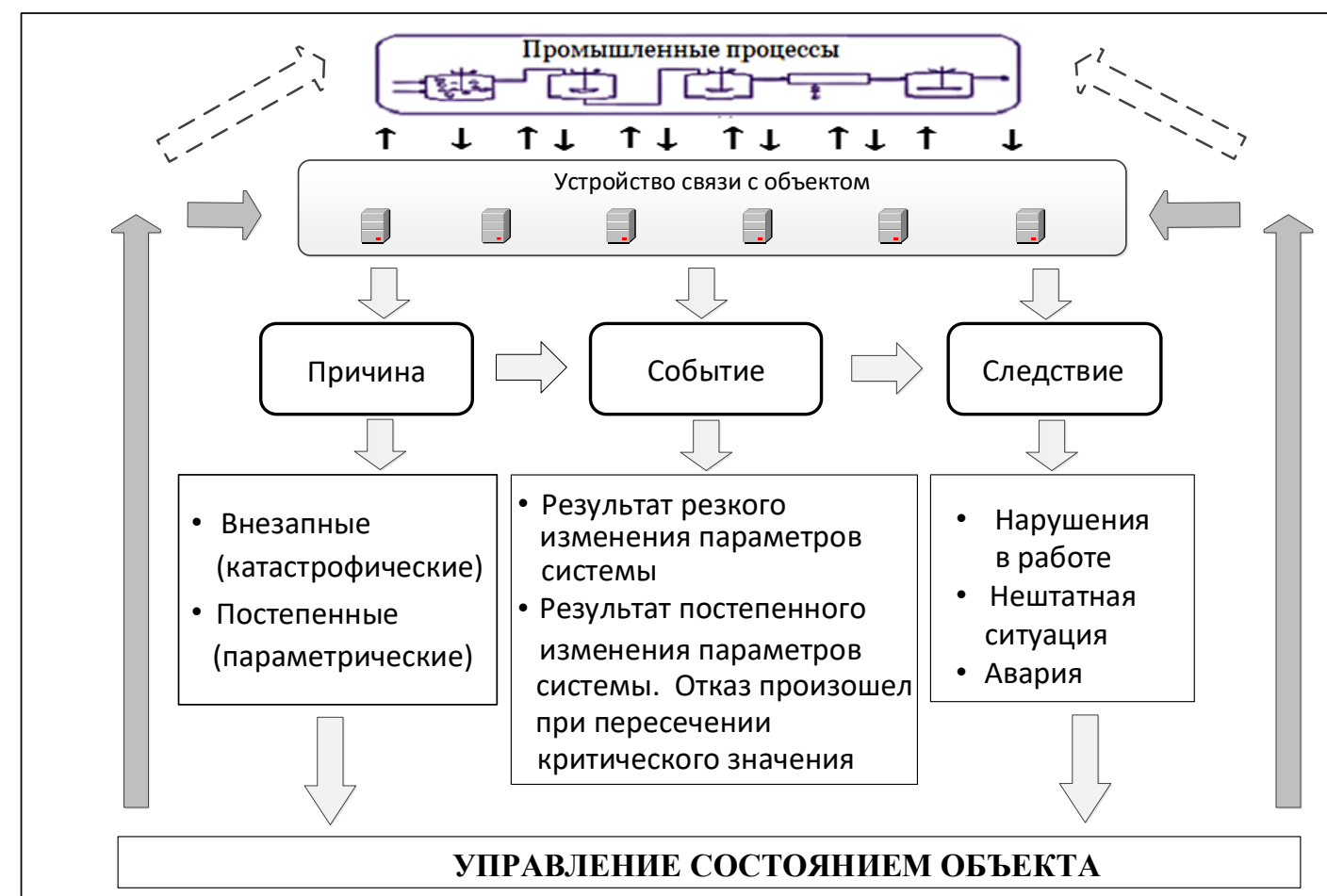

Рис. 1. Концептуальное представление системы управления технологической безопасностью

Fig. 1. Conceptual representation of technological safety management system

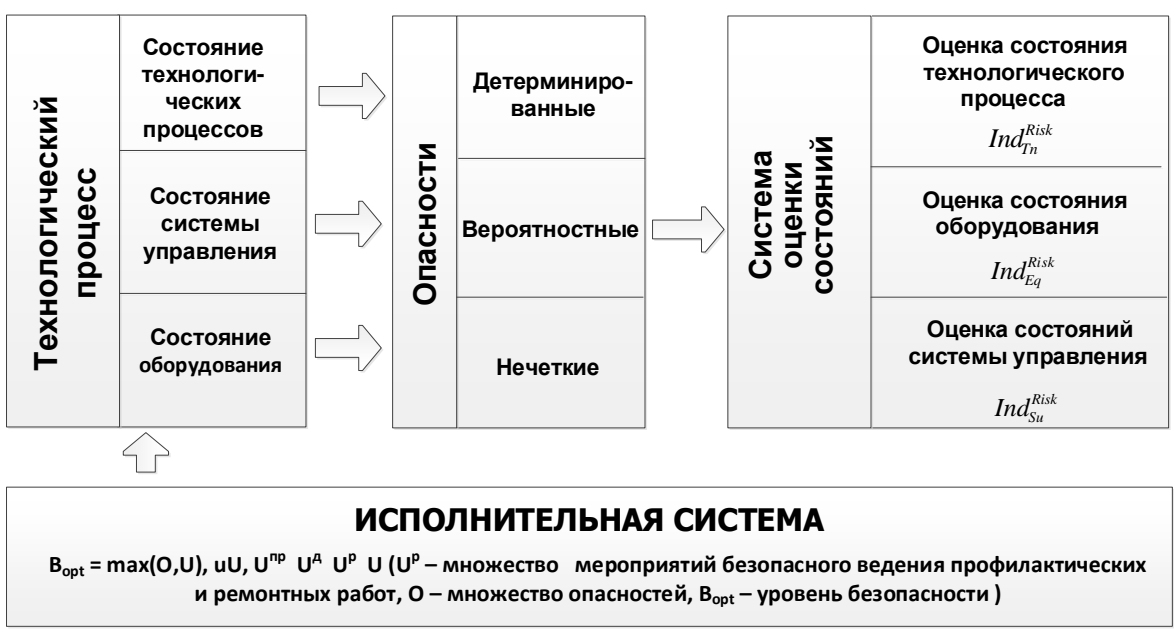

Рис. 2. Структура системы управления технологической безопасностью промышленньх процессов

Fig. 2. Structure of technological safety management system for industrial processes 
Существует ряд подходов к решению проблемы диагностирования состояний, например, дискретные модели [1], логико-вероятностные [2], причинно-следственные [3, 4], модели нечеткой логики [5], интервальные параметрические модели [6], байесовские [7], модели пространства состояний.

В большинстве случаев невозможно построить адекватную математическую модель управления технологической безопасностью в связи с тем, что критерии выбора при осуществлении процедур диагностики состояний промышленных технологий имеют нечеткий характер. Такая особенность задач приводит к необходимости использования экспертных оценок, которые зачастую служат единственной информацией для принятия решений, а также поиска методов для эффективной обработки полученной нечеткой экспертной информации. Примером такого подхода является метод разделения состояний, позволяющий осуществлять анализ состояний системы в условиях неопределенности информации об объекте.

Принципы построения модели диагностики состояний основаны на понятии области безопасного функционирования и центра безопасности диагностируемого объекта. Текущее состояние промышленных процессов оценивается по индексу безопасности, который содержательно характеризует степень удаленности текущего состояния процесса от центра безопасности. Количественно индекс безопасности $[1,6]$ определяется как пересечение области текущей ситуации и области центра безопасности: $v\left(\tilde{s}_{i}, \tilde{s}_{j}\right)=\underset{T}{\&} v\left(\mu_{s_{i}(T)}, \mu_{s_{j}(T)}\right)$,

где $v\left(\mu_{s_{i}(T)}, \mu_{s_{j}(T)}\right)=\underset{E_{k}}{\&}\left(\mu_{s_{i}(T)}\left(E_{k}\right) \rightarrow \mu_{s_{j}(T)}\left(E_{k}\right)\right)$,

$\mu_{\mu_{s_{i}(T)}\left(E_{k}\right) \rightarrow \mu_{s_{j}(T)}\left(E_{k}\right)}=\max \left\{1-\mu_{\mu_{s_{i}(T)}\left(E_{k}\right), \mu_{s_{j}(T)}\left(E_{k}\right)}\right\}$.

Для каждого из параметров в области их существования выделяются интервалы значений с целью определения области технологической безопасности функционирования технологии. Эти интервалы характеризуют определенные режимы работы промышленного процесса. Выбор недоминируемого интервала для области каждого параметра осуществляется на основе нечеткого отношения предпочтения во множестве альтернативных интервалов. В результате получается набор интервалов, отражающих состояния области работы технологии: $\Delta t^{1}, \Delta t^{2}$, $\ldots, \Delta t^{n}$. Здесь $\Delta t^{i}-$ множество интервалов $i$-го параметра. В каждом множестве $i$-го параметра выбираются интервал и точка в нем, характеризующие лучший режим по данному параметру. Точка $t_{0}^{i}$ определяет центр технологической безопасности $i$-го интервала. $S_{0}=\left\{\Delta t^{1} 0, \Delta t^{2}{ }_{0}, \ldots\right.$, $\left.\Delta t^{i} 0, \ldots, \Delta t^{n}{ }_{0}\right\}$ - область нормальной (исправной) работы. $\Delta t_{0}^{i}$ с некоторой нулевой точкой (центром) является благоприятным интервалом для $i$-го параметра.

Выявление центра технологической безопасности позволяет качественно и количественно оценить безопасные состояния технологии. Строится ситуационная таблица, которая возможной сложившейся ситуации на производстве ставит соответствующий набор управляющих воздействий. Для установления соответствия строятся нечеткие правила $\boldsymbol{D} \times \boldsymbol{U} \rightarrow \boldsymbol{R}$ выбора между ситуацией и набором управляющих воздействий. Для реализации нечеткого вывода вводится лингвистическая переменная по каждому параметру технологического процесса $\left\langle\beta_{i}, E_{i}, D_{i}>\right.$, где $\beta_{i}$ - название лингвистической переменной; $E_{i}=\left\{E_{i}^{1}, E_{i}^{2}, \ldots\right.$, $\left.E^{M i}{ }_{i}\right\}$ - терм-множество лингвистической переменной $\beta_{i} ; D_{i}-$ базовое множество лингвистической переменной $\beta_{i}$ (рис. 3).

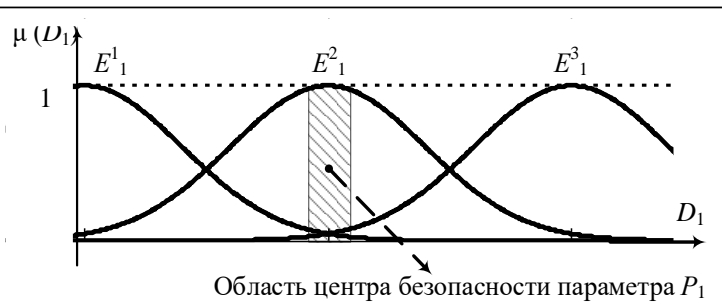

Рис. 3. Функиии принадлежности лингвистической переменной $<\beta_{i}, T_{i}, D_{i}>$

Fig. 3. Membership functions of the linguistic variable $\left\langle\beta_{i}, T_{i}, D_{i}\right\rangle$

Вектор, состоящий из множества лингвистических переменных $\beta_{i}$, нечетко определяет некоторое состояние технологии, характеризующее ситуацию.

Вводятся лингвистические переменные. Выполняется формализация семантической информации. Эта информация носит описательный, словесный характер, источником которой являются экспертные опросы обслуживающего персонала. Осуществляется перевод информации на язык нечеткой логики. Формируются управляющие решения. На основе мониторинговых измерений формируется БД (табл. 1) и вычисляются субъективные оценки идеала. 
Структура базы знаний с исходными данными и результатами счета

Knowledge base structure with initial data and calculation results

\begin{tabular}{|c|c|c|c|c|c|c|c|c|c|c|c|c|}
\hline & & $\begin{array}{r}\text { ходный } \\
\text { Сбор } \\
\text { Подго }\end{array}$ & $\begin{array}{l}\text { шаг нат } \\
\text { р инфор } \\
\text { отовител }\end{array}$ & $\begin{array}{l}\text { чала } \\
\text { мац } \\
\text { цьнь }\end{array}$ & $\begin{array}{l}\text { работь } \\
\text { ии по те } \\
\text { ге расче }\end{array}$ & $\begin{array}{l}\text { I программ } \\
\text { ехнологичес } \\
\text { еты функци }\end{array}$ & $\begin{array}{l}\text { иы систе } \\
\text { ским па } \\
\text { ий прин }\end{array}$ & $\begin{array}{l}\text { емы упI } \\
\text { араметр } \\
\text { Іадлежн }\end{array}$ & $\begin{array}{l}\text { рав. } \\
\text { оам. } \\
\text { Iост }\end{array}$ & тения. & & \\
\hline Номер & & $p_{1}$ & & $\ldots$ & & $P_{n}$ & $d$ & & $\ldots$ & & $d_{m}$ & \\
\hline состояния & $T_{1}$ & $T_{2}$ & $T_{3}$ & $\ldots$ & $T_{1}$ & $\ldots$ & $T_{1}$ & $T_{2}$ & $\ldots$ & $T_{1}$ & $T_{2}$ & $T_{3}$ \\
\hline 1 & $\mu_{11}\left(p_{1}\right)$ & $\mu_{12}\left(p_{1}\right)$ & $\mu_{13}\left(p_{1}\right)$ & $\ldots$ & $\mu_{11}\left(p_{n}\right)$ & $\mu_{1 k}\left(p_{n}\right)$ & $\mu_{11}\left(d_{1}\right)$ & $\mu_{12}\left(d_{1}\right)$ & $\ldots$ & $\mu_{1 n}\left(d_{m}\right)$ & $\mu_{12}\left(d_{m}\right)$ & $\mu_{13}\left(d_{m}\right)$ \\
\hline 2 & $\mu_{21}\left(p_{1}\right)$ & $\mu_{22}\left(p_{1}\right)$ & $\mu_{23}\left(p_{1}\right)$ & $\ldots$ & $\mu_{21}\left(p_{n}\right)$ & \begin{tabular}{l|l|}
$\ldots$ & $\mu_{2 k}\left(p_{n}\right)$ \\
\end{tabular} & $\mu_{21}\left(d_{1}\right)$ & $\mu_{22}\left(d_{1}\right)$ & $\ldots$ & $\mu_{21}\left(d_{m}\right)$ & $\mu_{22}\left(d_{m}\right)$ & $\mu_{23}\left(d_{m}\right)$ \\
\hline$\ldots$ & $\ldots$ & \begin{tabular}{|l|}
$\ldots$ \\
\end{tabular} & \begin{tabular}{|l|}
$\ldots$ \\
\end{tabular} & $\ldots$ & $\ldots$ & $\ldots$ & $\ldots$ & \begin{tabular}{|l|}
$\ldots$ \\
\end{tabular} & $\ldots$ & $\ldots$ & $\ldots$ & $\ldots$ \\
\hline$v$ & $\mu_{v 1}\left(p_{1}\right)$ & $\mu_{v 2}\left(p_{1}\right)$ & $\mu_{v 3}\left(p_{1}\right)$ & $\ldots$ & $\mu_{v 1}\left(p_{n}\right)$ & \begin{tabular}{l|l|}
$\ldots$ & $\mu_{v k}\left(p_{n}\right)$ \\
\end{tabular} & $\mu_{v 1}\left(p_{1}\right)$ & $\mu_{v 2}\left(p_{1}\right)$ & $\ldots$ & $\mu_{v 1}\left(d_{m}\right)$ & $\mu_{v 2}\left(d_{m}\right)$ & $\mu_{v 3}\left(d_{m}\right)$ \\
\hline & КорI & рекция & парамет & гров & модели & I- нечеткая & я нейро & сеть (A & NFI & S-сети) & & \\
\hline & & & & & & Этап 1 & & & & & & \\
\hline & $\begin{array}{r}\text { Pacy } \\
\text { In }\end{array}$ & $\begin{array}{l}\text { чет инде } \\
\imath\left(\tilde{S}_{p}^{*}\right), I\end{array}$ & $\begin{array}{l}\text { екса тех } \\
\operatorname{In}\left(\tilde{S}_{d}^{*}\right) \text { по }\end{array}$ & $\begin{array}{l}\text { нолс } \\
\text { отд }\end{array}$ & $\begin{array}{l}\text { गгическ } \\
\text { Іельным }\end{array}$ & $\begin{array}{l}\text { ой безопасн } \\
\text { м параметра }\end{array}$ & $\begin{array}{l}\text { ности и } \\
\text { ам (пра }\end{array}$ & $\begin{array}{l}\text { индекс } \\
\text { вило М }\end{array}$ & 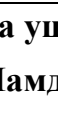 & $\begin{array}{l}\text { церба - } \\
\text { (ани) }\end{array}$ & & \\
\hline & & $p_{1}$ & & $\ldots$ & & $P_{n}$ & $d$ & & $\ldots$ & & $d_{m}$ & \\
\hline 1 & & $\operatorname{In}_{1}\left(\tilde{S}_{p 1}\right)$ & & $\cdots$ & & $1\left(\tilde{S}_{p n}\right)$ & $\operatorname{In}_{1}($ & $\left.\tilde{S}_{d 1}\right)$ & $\cdots$ & & $\operatorname{In}_{1}\left(\tilde{S}_{d m}\right)$ & \\
\hline$\ldots$ & & $\ldots$ & & $\ldots$ & & $\ldots$ & .. & & $\ldots$ & & $\ldots$ & \\
\hline$v$ & & $\operatorname{In}_{v}\left(\tilde{S}_{p 1}^{*}\right)$ & & $\cdots$ & & $v\left(\tilde{S}_{p n}^{*}\right)$ & $\operatorname{In}_{v}($ & $\left.\tilde{S}_{d 1}\right)$ & $\cdots$ & & $\operatorname{In}_{v}\left(\tilde{S}_{d m}\right)$ & \\
\hline & & & & & & Этап 2 & & & & & & \\
\hline & Pacu & чет инде & $\begin{array}{r}\text { екса тех } \\
\qquad \operatorname{In}\left(\tilde{S}_{p}^{*}\right)\end{array}$ & нолс & $\begin{array}{l}\text { огическ } \\
\left(\tilde{S}_{d}^{*}\right) \text { по }\end{array}$ & $\begin{array}{l}\text { ой безопасн } \\
\text { отдельным }\end{array}$ & $\begin{array}{l}\text { ности и } \\
\text { аппар: }\end{array}$ & $\begin{array}{l}\text { индекс } \\
\text { атам }\end{array}$ & a yu & дерба - & & \\
\hline 1 & & & $\operatorname{In}_{1}\left(\tilde{S}_{P}\right.$ & $(1-n)$ & & & & & $\operatorname{In}_{1}($ & $\left.\tilde{S}_{d(1-m)}\right)$ & & \\
\hline$\ldots$ & & & & & & & & & & $\ldots$ & & \\
\hline$v$ & & & $\operatorname{In}_{v}\left(\tilde{S}_{t}\right.$ & $(1-n)$ & & & & & $I n_{v}$ & $\left(\tilde{S}_{d(1-n)}\right)$ & & \\
\hline
\end{tabular}

Расчет индекса риска - $\operatorname{In}_{R i s k}\left(\tilde{S}^{*}\right)=\left\{\operatorname{In}\left(\tilde{S}_{p}^{*}\right), \operatorname{In}\left(\tilde{S}_{d}^{*}\right)\right\}$ в дискретные моменты времени

\begin{tabular}{|c|c|}
\hline 1 & $\operatorname{In}_{\text {Risk } 1}(\tilde{S})$ \\
\hline$\ldots$ & $\ldots$ \\
\hline$v$ & $\operatorname{In}_{\text {Riskv }}(\tilde{S})$ \\
\hline
\end{tabular}

Принятие решений - выбор изменений нагрузки на технологию селективной очистки по аммиаку и добавочному воздуху. Регулирование на основе прогнозирующих моделей МРС-контроллеры

Определение нагрузки на систему по аммиаку и добавочному воздуху (правило Мамдани)

Переход на исходный шаг начала работы программы системы управления процессом

Структура БД отражает варианты расчета для показателей безопасности функционирования технологической системы. При этом учитывается ущерб, возникающий в процессе функционировании системы. На основе того же механизма вычислений определяется индекс субъективной оценки риска:

$$
\operatorname{In}_{\text {Risk }}\left(\tilde{S}^{*}\right)=\left\{\operatorname{In}\left(\tilde{S}_{p}^{*}\right), \operatorname{In}\left(\tilde{S}_{d}^{*}\right)\right\}
$$

где $\operatorname{In}\left(\tilde{S}_{p}^{*}\right)$ - индекс технологической безопасности; In $\left(\tilde{S}_{d}^{*}\right)$ - индекс ущерба [1].

В работе рассмотрено создание системы управления технологическим процессом селективной очистки газов при производстве неконцентрированной азотной кислоты на основе моделирования возможных ситуаций. Созда- 
ние системы управления химико-технологическим процессом селективной очистки газов при производстве неконцентрированной азотной кислоты предусматривает учет влияния случайных возмущений, что приводит к возникновению различных ситуаций при работе технологического процесса [8]. Функциональная структура системы управления технологической безопасностью представлена на рисунке 4.

\section{Характеристика системы управления на основе прогнозирующих моделей систем управления}

В работе предлагается на основе оценки уровня риска работы промышленной технологии диагностировать состояние процесса, после чего строить многоуровневую систему управления промышленным процессом. Для оценки риска используется нечетко определенная импульсная модель риска, которая учитывает возможные потери от внезапных и постепенных отказов, что повышает эффективность функционирования системы управления. На основе оценки состояния технологической системы определяется нагрузка системы и осуществляется коррекция параметров математической модели (рис. 4).

Одним из перспективных направлений повышения качества работы систем управления является использование семейства МРС-контроллеров (системы управления с прогнозирующими моделями), которые реализуют управление на основе предсказания параметров, определяющих состояние процесса [8, 9]. Параметры системы:

- входные значения процесса - заданные значения центра технологической безопасности $r_{k}$;
- выходные значения процесса $z$ - измеренные выходы процесса $y$;

- регулирующие переменные $-u$.

Управляющие переменные:

- количество восстановителя в реакторе очистки, определяемое подачей аммиака $\left(G_{N_{3}}\right)$ в реактор;

- концентрация кислорода перед реактором очистки, а значит, степень конверсии аммиака в реакторе, определяемая подачей доба-

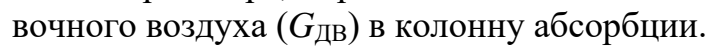

Регулируемые параметры:

- температура газа на входе $T^{\text {вx. }}$ в объект (реактор);

- температура на выходе $T^{\text {вых. }}$ из объекта (реактора);

- концентрация окислов азота $\left(C_{\mathrm{NO}_{\mathrm{NO}}}\right)$.

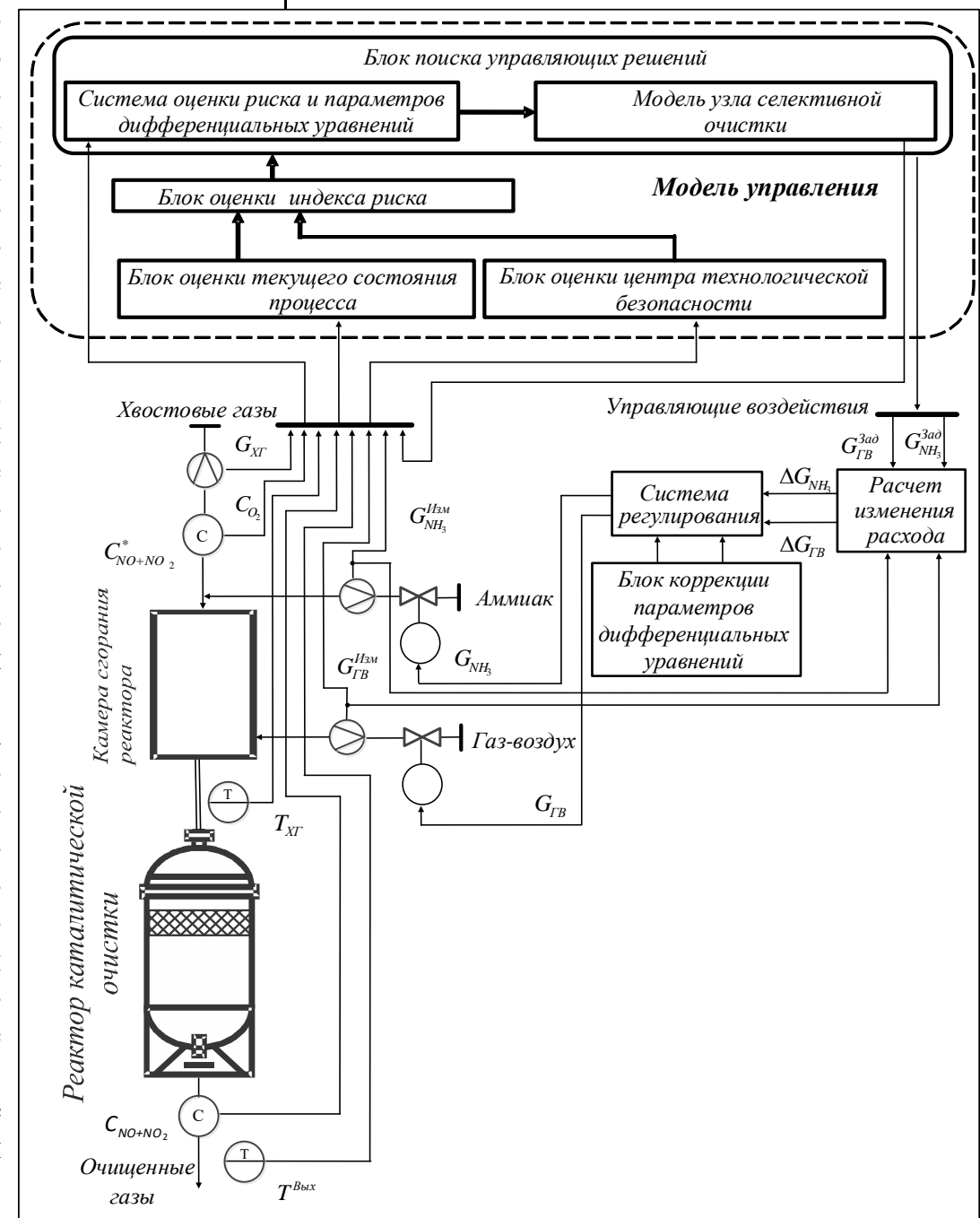

Рис. 4. Функциональная структура системы управления реактором селективной очистки

Fig. 4. Functional structure of the selective reactor control system 
Возмущения - рассчитанные значения рисков по состояниям оборудования, системы управления, технологии.

Опишем механизмы построения нечетко определенной импульсной модели оценки состояния системы и движения системы к точке безопасности. На рисунке 5 представлены графическая иллюстрация центра безопасности и формирование функции индекса безопасности относительно установленного центра.

На рисунке 6 графически проиллюстрированы индекс безопасности Ind $\left(z_{k+1}\right)$ - индекс по контролируемому параметру, а также значение Ind $^{z d}\left(r_{k+1}\right)$ - величина заданного значения индекса желаемого значения параметра.

На рисунке 7 представлен график технологического параметра, а также приводятся гра-

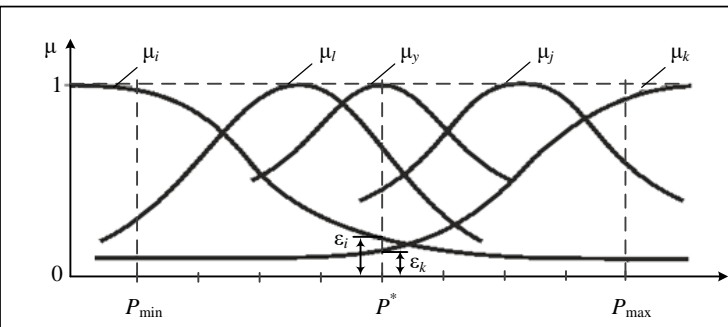

Рис. 5. Возможный вид функииий принадлежности параметра прочесса к различным состояниям

Fig. 5. Possible form of membership functions of a process parameter to various states

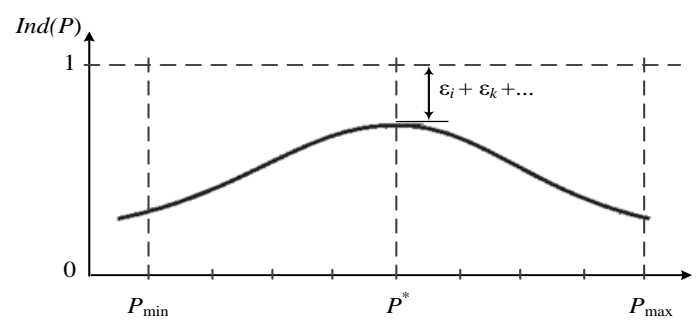

Рис. 6. Возможный вид графика индекса безопасности

Fig. 6. Possible view of safety index chart

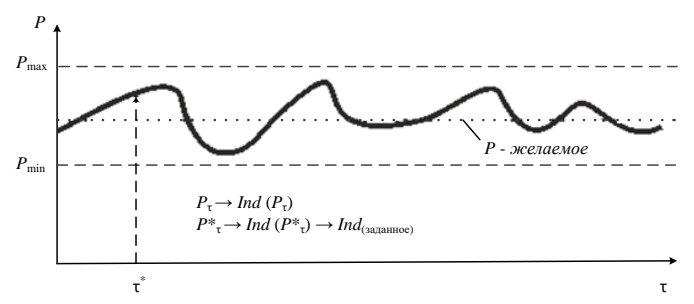

Рис. 7. График текущих значений технологического параметра

Fig. 7. The chart of current values for a technological parameter ничные значения параметра и наилучшее значение в данной технологической ситуации $\left(P_{\text {жел }}^{*}\right)$.

График иллюстрирует текущее отклонение параметра от наилучшего значения. На основе этого отклонения определяются регулирующие значения управляющих воздействий: подача аммиака в реактор $\left(G_{N_{3}}\right)$ и подача добавочного воздуха $\left(G_{\text {дв}}\right)$ в колонну абсорбции. Удаленность мгновенного значения параметра $P$ от наилучшего (желаемого) значения параметра $P^{*}$ - центра технологической безопасности - формирует индекс безопасности технологического процесса. За центр берется максимальное $P^{*} \in\left[P_{\min }, P_{\max }\right]$, а за ноль координаты $P_{\min }$ и $P_{\max }$, где значение индекса безопасности близко к нулю.

На рисунке 8 показан механизм формирования импульсов на определенном интервале времени.

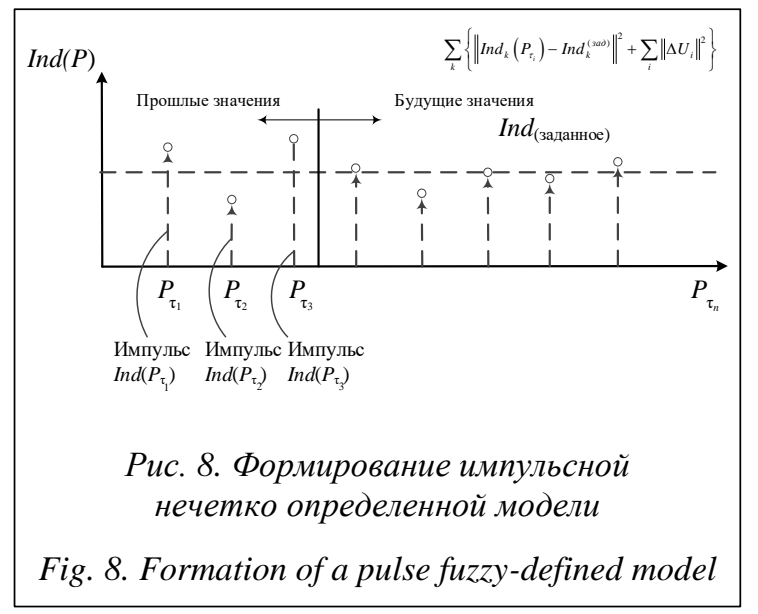

Авторами разработана модель системы управления процессом селективной очистки газов с подсистемой оценки параметров дифференциальных уравнений модели (см. http:// www.swsys.ru/uploaded/image/2019-4/2019-4dop/20.jpg), алгоритм работы которой, построенный в программной среде MATLAB, представлен на рисунке 9.

\section{Обобщенный алгоритм работы системы}

1. Собираются данные с объекта.

2. Осуществляется коррекция точки центра безопасности.

3. Назначается Ind ${ }^{z d}\left(r_{k+1}\right)$ - задание для регулятора в соответствии с режимом работы.

4. Обновляется импульсная нечеткая модель. 
5. Осуществляется шаг в направлении Ind $^{z d}\left(r_{k+1}\right)$, и по переменным состояния определяется индекс технологической безопасности для прогнозного $k+1$ интервала времени $\operatorname{Ind}\left(z_{k+1}\right)$.

6. Выполняется запись индексов безопасности в БД для последующей диагностики состояния процесса и его оборудования.
7. Если изменение временного ряда стационарно, осуществляется выдача управляющих воздействий (рис. 9).

8. Если изменение временного ряда нестационарно, выполняется диагностика состояния и принимаются решения по устранению возникшего нарушения (рис. 9).

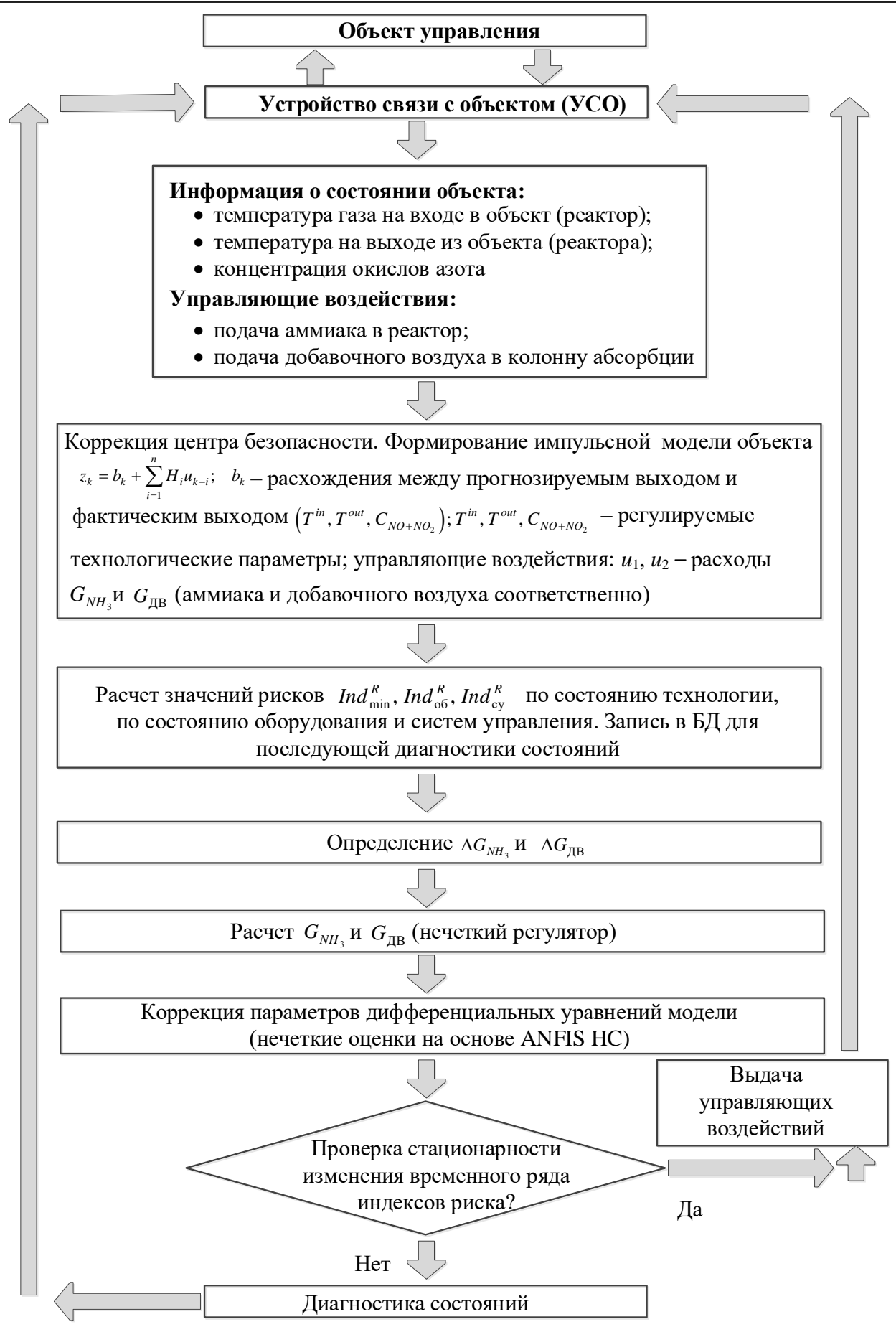

Рис. 9. Алгоритм работы системы управления объекта

Fig. 9. The working algorithm of the facility control system 
АСУ ТП обеспечивает получение информации, ее передачу в систему обработки данных, саму обработку, систематизацию, ранжирование данных, определение управляющих воздействий технологическим процессом, регламентирует оперативные действия (в том числе и с участием персонала), выдачу отчетов, информационных сообщений и рекомендаций. В работе были рассмотрены несколько вариантов систем управления и приведен лучший вариант по показателям качества.

В системе управления реализованы три уровня управления. Первый уровень - коррекция коэффициентов моделей по каналам связи технологических переменных (блоки нечеткой коррекции). Второй уровень - определение нагрузки на реактор по подаче аммиака (нечеткое регулирование), а также коррекция подачи воздуха в реактор - два МРС-регулятора. Третий уровень - определение изменения нагрузки на систему по аммиаку и воздуху в зависимости от индекса риска работы технологии (два МРС-регулятора).

Проверка модели на адекватность и качество работы осуществлялась в программной среде MATLAB методом статистического моделирования с использованием информации из технической документации реального производства (см. http://www.swsys.ru/uploaded/image/2019-4/2019-4-dop/18.jpg). По полученным данным определялось распределение входных и выходных переменных, а также осуществлялось сравнение значений рассчитанных по каждому параметру характеристик старой и новой систем управления.

Функции распределения «Концентрация хвостовых газов у выхода реактора» и «Температура у выхода реактора селективной очистки газов» представлены на рисунке (см. http:// www.swsys.ru/uploaded/image/2019-4/2019-4dop/19.jpg).

Для полученных данных определялись гистограммы, математическое ожидание, дисперсия температуры хвостовых газов и концентрации окислов азота.

Сопоставляя полученные результаты с данными существующей системы управления, можно сделать вывод, что дисперсия регулируемой величины значительно снизилась.

Аналогично вычисляются математическое ожидание и дисперсия концентрации хвостовых газов у выхода реактора.

При сопоставлении экспериментальных (по работе действующей модели) и модельных данных можно сделать однозначный вывод об адекватной работе новой модели управления.

\section{Обоснование применения разработанной системы управления селективной очисткой газов}

На основе анализа действующих промышленных технологических агрегатов показано, что оптимальное управление ими может быть осуществлено только с учетом наиболее полного использования современных систем управления - в частности, интеллектуальных МРС-регуляторов.

Анализ дисперсий для основных текущих технологических параметров, на основе которых определяется индекс риска (табл. 2), показал, что изменение дисперсии в рабочем цикле по температуре составляет порядка 15-20\%, по концентрации - около $20 \%$.

Так как дисперсия характеризует разброс параметров, можно сделать вывод: аналогичный разброс будет сопровождать процесс селективной очистки и по нечетким индексам риска. Учитывая, что динамика изменения технологических параметров выше динамики изменения надежности оборудования и систем управления, можно сделать вывод о росте стабильности работы системы для текущих режимов работы примерно на 10-20\%.

Таблица 2

Результаты вычислительного эксперимента Table 2

Computational experiment results

\begin{tabular}{|c|c|c|}
\hline Система & $\begin{array}{c}\text { Темп } \\
\text { после }\end{array}$ & or \\
\hline 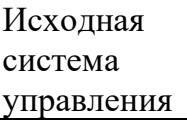 & $\begin{array}{l}=276 \\
=35\end{array}$ & $\begin{array}{l}M(x)=0.0021 ; \\
D(x)=0.00014\end{array}$ \\
\hline & & \\
\hline & $\begin{array}{c}1-20,0 / 35,0= \\
=0,17\end{array}$ & $\begin{array}{c}1-0,011 / 0,014= \\
=0,21\end{array}$ \\
\hline
\end{tabular}

Индекс риска по оборудованию и системам управления определяется для оценки состояний процесса селективной очистки, а также принятия решений по проведению текущих профилактических осмотров и ремонтов.

Построена математическая нечетко определенная модель реактора селективной очистки, отражающая основные фундаментальные свойства процесса, воспринимаемые человеком и существующие в агрегате. Проведено моделирование этих соотношений, определены технологические характеристики агрегата. Произведена оценка влияния реальных возмущающих воздействий на показатели качества управления. 
Сделан вывод, что для улучшения показателей качества управления реактором селективной очистки хвостовых газов необходимы разработка и реализация системы автоматического регулирования разности температур в реакторе каталитической очистки, построенной на основании нетиповых законов регулирования. В результате исследований для регулирования температурного режима в реакторе и координации подачи воздуха и аммиака предложено использовать регулятор с предсказа- нием, для определения задания по воздуху и аммиаку - нечеткий регулятор, для повышения точности модели - динамическую коррекцию параметров дифференциальных уравнений.

Разработанная система автоматического регулирования узла каталитической очистки позволила не только повысить экономические показатели работы цеха, но и улучшить очистку хвостовых газов от окислов азота и, следовательно, уменьшить загрязнение воздушного бассейна.

Работа выполнена при финансовой поддержке РФФИ, грант № 17-07-013680.

\title{
Лumepamypa
}

1. Вент Д.П., Лопатин А.Г., Брыков Б.А., Богатиков В.Н., Пророков А.Е. Диагностика состояний и управление динамическими процессами в слабоструктурированных и плохоформализуемых средах. Новомосковск: Изд-во НИ РХТУ, 2018. 199 с.

2. Solozhentsev E.D. Risk management technologies with logic and probabilistic models. Springer, 2012, $328 \mathrm{p}$.

3. Науменко А.П. Методы технической диагностики. Омск: Изд-во ОмГТУ, 2016. 125 с.

4. Бурлаченко Т.Б. Методы и алгоритмы интеллектуальной поддержки принятия решений в задачах выявления причинно-следственных связей и диагностики технических систем, используемых в машиностроении. М.: Изд-во МГУПИ, 2009. 149 с.

5. Яхъяева Г.Э. Нечеткие множества и нейронные сети. М.: ИНТУИТ, 2014. 320 с.

6. Палюх Б.В., Богатиков В.Н., Пророков А.Е., Алексеев В.В. Приложение метода разделения состояний для управления технологической безопасностью промышленных процессов на основе нечетко определенных моделей. Тверь: Изд-во ТГТУ, 2009. 348 с.

7. Дауни А.Б. Байесовские модели. Байесовская статистика на языке Python. ДМК-Пресс, 2018. $182 \mathrm{c}$.

8. Веремей Е.И., Сотникова М.В. Управление с прогнозирующими моделями. СПб: Изд-во СПбГУ, 2014. 212 c.

9. Bemporad A., Morari M., Ricke N.L. Model Predictive Control Toolbox ${ }^{\mathrm{TM}}$ Getting Started Guide. URL: https://fumblog.um.ac.ir/gallery/839/mpc_gs.pdf (дата обращения: 05.08.2019).

Software \& Systems

DOI: $10.15827 / 0236-235 X .128 .725-734$
Received 09.08.19

2019 , vol. 32 , no. 4 , pp. $725-734$

\section{Industrial technologies states management based on risk criterion}

\author{
S.R. Bakasov ${ }^{1,}$ Postgraduate Student, sabir17204@gmail.com \\ G.N. Sanaeva ${ }^{2}$, Senior Lecturer, gsanaeva@nirhtu.ru \\ V.N. Bogatikov ${ }^{1}$, Dr.Sc. (Engineering), Professor,vnbgtk@mail.com \\ ${ }^{1}$ Tver State Technical University, Tver, 170026, Russian Federation \\ ${ }^{2}$ Novomoskovsky Institute of the D. Mendeleev University of Chemical Technology of Russian Federation, \\ Novomoskovsk, 301665, Russian Federation
}

Abstract. The present paper is devoted to the study of conceptual formulation of the industrial technologies state management problem. It considers managing a potentially hazardous technology for selective treatment of tail gases from production of non-concentrated nitric acid. This is a practical application of the state management idea. In this application state management is closely connected with industrial systems safety management. 
One of the problems in the synthesis of industrial technologies safety management systems is the presence of uncertainty both in the knowledge of physicochemical processes and in the uncertainty associated with the influence of random disturbances. This gives rise to the development of new methods for the synthesis of technological safety management systems, as well as to the improvement of existing ones. As a consequence of the above factors, methods for implementing goal-setting mechanisms and revising control quality criteria become a promising approach for this kind of dynamic processes occurring in poorly structured and poorly formalized environments. These methods rest upon fundamental background knowledge. Various types of defects are reflected in technological processes state variables. Violations can be caused by defects in control systems, process equipment and violations in the technological process itself. All kinds of damage in the technological system (non-compliance with the requirements of source materials, non-compliance with the requirements of regulatory and technical documents and the human factor) lead to similar results. This indicates both the complexity of diagnosing procedure and the complexity of forming criteria to assess states.

At the present time from a management point of view, a technological safety system is a multi-level hierarchically organized technological system. The main goals of such systems are to timely detect malfunctions and to take measures to eliminate their root causes. The paper considers multi-level organization of the technological safety system.

As a practical application of the proposed approach the paper considers multi-level organization of the technological safety system for the process of selective treatment of tail gases from the production of weak nitric acid. Authors propose the main technology management criterion (a risk criterion for conducting the technological process) and the impulse model of the criterion. Management is based on predictive management.

The developed system allowed not only to increase economic indicators, but also to reduce air pollution.

Keywords: state diagnosing, providing technological safety, industrial technology risk.

Acknowledgements. The paper was financially supported by the Russian Foundation for Basic Research (RFBR), grant no. 17-07-013680.

\section{References}

1. Vent D.P., Lopatin A.G., Brykov B.A., Bogatikov V.N., Prorokov A.E. Diagnostics of States and Control of Dynamic Processes in the Semistructured and Badly Formalizable Environments. Novomoskovsk, 2018, 199 p. (in Russ.).

2. Solozhentsev E.D. Risk Management Technologies with Logic and Probabilistic Models. Springer, 2012, 328 p.

3. Naumenko A.P. Technical Diagnostic Methods. Omsk, OmSTU Publ., 2016, 125 p. (in Russ.).

4. Burlachenko T.B. Methods and Algorithms of Intellectual Decision-Making Support in the Tasks of Identifying Cause-Effect Relationships and Diagnostics of Technical Systems Used in Mechanical Engineering. Moscow, 2009, 149 p. (in Russ.).

5. Yakhyaeva G.E. Fuzzy Sets and Neural Networks. Moscow, Intuit Publ., 2014, 320 p. (in Russ.).

6. Palyukh B.V. An Application of the State Separation Method for Controlling the Technological Safety of Industrial Processes Based on Fuzzy Defined Models. Tver, TSTU Publ., 2009, 348 p. (in Russ.).

7. Dauni A. Bayesian Models. Bayesian Statistics in Python. O'Reilly Publ., 2013. Russ. ed.: DMK-Press, 2018, 182 p.

8. Veremey E.I., Sotnikova M.V. Predictive Model Management. St. Petersburg, SPBU Publ., 2014, 212 p. (in Russ.).

9. Bemporad A., Morari M., Ricke N.L. Model Predictive Control Toolbox ${ }^{\mathrm{TM}}$ Getting Started Guide. Available at: https://fumblog.um.ac.ir/gallery/839/mpc_gs.pdf (accessed September 05, 2019).

\section{Для цитирования}

Бакасов С.Р., Санаева Г.Н., Богатиков В.Н. Управление состояниями промышленных технологий на основе критерия риска // Программные продукты и системы. 2019. Т. 32 . № 4. C. 725-734. DOI: 10.15827/0236-235X.128.725-734.

\section{For citation}

Bakasov S.R., Sanayeva G.N., Bogatikov V.N. Industrial technologies states management based on risk criterion. Software \& Systems. 2019, vol. 32, no. 4, pp. 725-734 (in Russ.). DOI: 10.15827/0236-235X.128.725-734. 